CUBO A Mathematical Journal

Vol.21, № 03, (29-38). December 2019

\title{
Ostrowski-Sugeno fuzzy inequalities
}

\author{
George A. Anastassiou \\ Department of Mathematical Sciences \\ University of Memphis \\ Memphis, TN 38152, U.S.A. \\ ganastss@memphis.edu
}

\begin{abstract}
We present Ostrowski-Sugeno fuzzy type inequalities. These are Ostrowski-like inequalities in the context of Sugeno fuzzy integral and its special properties are investigated. Tight upper bounds to the deviation of a function from its Sugeno-fuzzy averages are given. This work is greatly inspired by [3] and [1].

\section{RESUMEN}

Presentamos desigualdades de Ostrowski-Sugeno de tipo fuzzy. Estas son desigualdades de tipo Ostrowski en el contexto de integrales fuzzy de Sugeno y se investigan sus propiedades especiales. Se entregan cotas superiores ajustadas para la desviación de una función de sus promedios fuzzy de Sugeno. Este trabajo está inspirado principalmente por [3] y [1].
\end{abstract}

Keywords and Phrases: Sugeno fuzzy, integral, function fuzzy average, deviation from fuzzy mean, fuzzy Ostrowski inequality.

2010 AMS Mathematics Subject Classification: Primary: 26D07, 26D10, 26D15, 41A44, Secondary: 26A24, 26D20, 28A25. 


\section{Introduction}

The famous Ostrowski ([3]) inequality motivates this work and has as follows:

$$
\left|\frac{1}{b-a} \int_{a}^{b} f(y) d y-f(x)\right| \leq\left(\frac{1}{4}+\frac{\left(x-\frac{a+b}{2}\right)^{2}}{(b-a)^{2}}\right)(b-a)\left\|f^{\prime}\right\|_{\infty},
$$

where $f \in C^{\prime}([a, b]), x \in[a, b]$, and it is a sharp inequality. One can easily notice that

$$
\left(\frac{1}{4}+\frac{\left(x-\frac{a+b}{2}\right)^{2}}{(b-a)^{2}}\right)(b-a)=\frac{(x-a)^{2}+(b-x)^{2}}{2(b-a)} .
$$

Another motivation is author's article [1].

First we give a survey about Sugeno fuzzy integral and its basic properties. Then we derive a series of Ostrowski-like inequalities to all directions in the context of Sugeno integral and its basic important particular properties. We also give applications to special cases of our problem we deal with.

\section{Background}

In this section, some definitions and basic important properties of the Sugeno integral which will be used in the next section are presented.

Definition 2.1. (Fuzzy measure [5, 27]) Let $\Sigma$ be a $\sigma$-algebra of subsets of $\mathrm{X}$, and let $\mu: \Sigma \rightarrow[0,+\infty]$ be a non-negative extended real-valued set function. We say that $\mu$ is a fuzzy measure iff:

(1) $\mu(\varnothing)=0$,

(2) $\mathrm{E}, \mathrm{F} \in \Sigma: \mathrm{E} \subseteq \mathrm{F}$ imply $\mu(\mathrm{E}) \leq \mu(\mathrm{F})$ (monotonicity),

(3) $E_{n} \in \Sigma(n \in \mathbb{N}), E_{1} \subset E_{2} \subset \ldots$, imply $\lim _{n \rightarrow \infty} \mu\left(E_{n}\right)=\mu\left(\cup_{n=1}^{\infty} E_{n}\right)$ (continuity from below);

(4) $E_{n} \in \Sigma(n \in \mathbb{N}), E_{1} \supset E_{2} \supset \ldots, \mu\left(E_{1}\right)<\infty$, imply $\lim _{n \rightarrow \infty} \mu\left(E_{n}\right)=\mu\left(\cap_{n=1}^{\infty} E_{n}\right)$ (continuity from above).

Let $(X, \Sigma, \mu)$ be a fuzzy measure space and $f$ be a non-negative real-valued function on $X$. We denote by $\mathcal{F}_{+}$the set of all non-negative real valued measurable functions, and by $L_{\alpha} f$ the set: $L_{\alpha} f:=\{x \in X: f(x) \geq \alpha\}$, the $\alpha$-level of $f$ for $\alpha \geq 0$.

Definition 2.2. Let $(X, \Sigma, \mu)$ be a fuzzy measure space. If $\mathrm{f} \in \mathcal{F}_{+}$and $\mathrm{A} \in \Sigma$, then the Sugeno integral (fuzzy integral) [6] of $\mathrm{f}$ on $\mathrm{A}$ with respect to the fuzzy measure $\mu$ is defined by

$$
\text { (S) } \int_{A} f d \mu:=\vee_{\alpha \geq 0}\left(\alpha \wedge \mu\left(A \cap L_{\alpha} f\right)\right),
$$

where $\vee$ and $\wedge$ denote the sup and inf on $[0, \infty]$, respectively. 
The basic properties of Sugeno integral follow:

Theorem 2.3. (4, 7]) Let $(\mathrm{X}, \Sigma, \mu)$ be a fuzzy measure space with $\mathrm{A}, \mathrm{B} \in \Sigma$ and $\mathrm{f}, \mathrm{g} \in \mathcal{F}_{+}$. Then

1) (S) $\int_{A} f d \mu \leq \mu(A)$;

2) (S) $\int_{A} k d \mu=k \wedge \mu(A)$ for a non-negative constant $k$;

3) if $\mathrm{f} \leq \mathrm{g}$ on $\mathrm{A}$, then $(\mathrm{S}) \int_{\mathrm{A}} \mathrm{fd} \mu \leq(\mathrm{S}) \int_{\mathrm{A}} \mathrm{gd} \mu$;

4) if $A \subset B$, then $(S) \int_{A} f d \mu \leq(S) \int_{B} f d \mu$;

5) $\mu\left(A \cap L_{\alpha} f\right) \leq \alpha \Rightarrow(S) \int_{A} f d \mu \leq \alpha$;

6) if $\mu(A)<\infty$, then $\mu\left(A \cap \mathrm{L}_{\alpha} f\right) \geq \alpha \Leftrightarrow(S) \int_{A} f d \mu \geq \alpha$;

7) when $A=X$, (S) $\int_{A} f d \mu=V_{\alpha \geq 0}\left(\alpha \wedge \mu\left(L_{\alpha} f\right)\right)$;

8) if $\alpha \leq \beta$, then $\mathrm{L}_{\beta} \mathrm{f} \subseteq \mathrm{L}_{\alpha} \mathrm{f}$;

g) (S) $\int_{A} f d \mu \geq 0$.

Theorem 2.4. ([7, p. 135]) Let $\mathrm{f} \in \mathcal{F}_{+}$, the class of all finite nonnegative measurable functions on $(X, \Sigma, \mu)$. Then

1) if $\mu(A)=0$, then $(S) \int_{A} f d \mu=0$, for any $f \in \mathcal{F}_{+}$;

2) if (S) $\int_{A} f d \mu=0$, then $\mu(A \cap\{x \mid f(x)>0\})=0$;

3) $(S) \int_{A} f d \mu=(S) \int_{A} f \cdot \chi_{A} d \mu$, where $\chi_{A}$ is the characteristic function of $A$;

4) (S) $\int_{A}(f+a) d \mu \leq(S) \int_{A} f d \mu+(S) \int_{A} a d \mu$, for any constant $a \in[0, \infty)$.

Corollary 2.5. ([7, p. 136]) Let $\mathrm{f}, \mathrm{f}_{1}, \mathrm{f}_{2} \in \mathcal{F}_{+}$. Then

1) (S) $\int_{A}\left(f_{1} \vee f_{2}\right) d \mu \geq(S) \int_{A} f_{1} d \mu \vee(S) \int_{A} f_{2} d \mu$;

2) $(S) \int_{A}\left(f_{1} \wedge f_{2}\right) d \mu \leq(S) \int_{A} f_{1} d \mu \wedge(S) \int_{A} f_{2} d \mu$;

3) (S) $\int_{A \cup B} f d \mu \geq(S) \int_{A} f d \mu \vee(S) \int_{B} f d \mu$;

4) (S) $\int_{A \cap B} f d \mu \leq(S) \int_{A} f d \mu \wedge(S) \int_{B} f d \mu$.

In general we have

$(S) \int_{A}\left(f_{1}+f_{2}\right) d \mu \neq(S) \int_{A} f_{1} d \mu+(S) \int_{A} f_{2} d \mu$,

and

(S) $\int_{A} a f d \mu \neq a(S) \int_{A} f d \mu$, where $a \in \mathbb{R}$,

see [7, p. 137].

Lemma 2.6. ([7, p. 138]) (S) $\int_{A} f d \mu=\infty$ if and only if $\mu\left(A \cap \mathrm{L}_{\alpha} f\right)=\infty$ for any $\alpha \in[0, \infty)$.

We need 
Definition 2.7. ([2]) A fuzzy measure $\mu$ is subadditive iff $\mu(A \cup B) \leq \mu(A)+\mu(B)$, for all $A, B \in \Sigma$.

We mention the following result

Theorem 2.8. ([2]) If $\mu$ is subadditive, then

$$
(S) \int_{X}(f+g) d \mu \leq(S) \int_{X} f d \mu+(S) \int_{X} g d \mu
$$

for all measurable functions $\mathrm{f}, \mathrm{g}: \mathrm{X} \rightarrow[0, \infty)$.

Moreover, if (2) holds for all measurable functions $\mathrm{f}, \mathrm{g}: \mathrm{X} \rightarrow[0, \infty)$ and $\mu(\mathrm{X})<\infty$, then $\mu$ is subadditive.

Notice here in (1) we have that $\alpha \in[0, \infty)$.

We have the following corollary.

Corollary 2.9. If $\mu$ is aubadditive, $\mathrm{n} \in \mathbb{N}$, and $\mathrm{f}: \mathrm{X} \rightarrow[0, \infty)$ is a measurable function, then

$$
\text { (S) } \int_{X} n f d \mu \leq n(S) \int_{X} f d \mu,
$$

in particular it holds

$$
(S) \int_{A} n f d \mu \leq n(S) \int_{A} f d \mu
$$

for any $\mathrm{A} \in \Sigma$.

Proof. By inequality (2).

A very important property of Sugeno integral follows.

Theorem 2.10. If $\mu$ is subadditive measure, and $\mathrm{f}: \mathrm{X} \rightarrow[0, \infty)$ is a measurable function, and $c>0$, then

$$
(S) \int_{A} c f d \mu \leq(c+1)(S) \int_{A} f d \mu
$$

for any $\mathrm{A} \in \Sigma$.

Proof. Let the ceiling $\lceil\mathrm{c}\rceil=\mathrm{m} \in \mathbb{N}$, then by Theorem 2.3 (3) and (4) we get

$$
(S) \int_{A} \operatorname{cfd} \mu \leq(S) \int_{A} m f d \mu \leq m(S) \int_{A} f d \mu \leq(c+1)(S) \int_{A} f d \mu,
$$

proving (5). 


\section{Main Results}

From now on in this article we work on the fuzzy measure space $([a, b], \mathcal{B}, \mu)$, where $[a, b] \subset \mathbb{R}, \mathcal{B}$ is the Borel $\sigma$-algebra on $[a, b]$, and $\mu$ is a finite fuzzy measure on $\mathcal{B}$. Typically we take it to be subadditive.

The functions $f$ we deal with here are continuous from $[a, b]$ into $\mathbb{R}_{+}$.

We make the following remark

Remark 3.1. Let $\mathrm{f} \in \mathrm{C}^{1}\left([\mathrm{a}, \mathrm{b}], \mathbb{R}_{+}\right)$, and $\mu$ is a subadditive fuzzy measure such that $\mu([\mathrm{a}, \mathrm{b}])>0$, $x \in[\mathrm{a}, \mathrm{b}]$. We will estimate

$$
E:=\left|(S) \int_{[a, b]} f(x) d \mu(t)-\mu([a, b]) \wedge f(x)\right|
$$

(by Theorem 2.3 (2))

$$
=\left|(S) \int_{[a, b]} f(t) d \mu(t)-(S) \int_{[a, b]} f(x) d \mu(t)\right| .
$$

We notice that

$$
f(t)=f(t)-f(x)+f(x) \leq|f(t)-f(x)|+f(x),
$$

then (by Theorem 2.3 (3) and Theorem 2.4 (4))

$$
(S) \int_{[a, b]} f(t) d \mu(t) \leq(S) \int_{[a, b]}|f(t)-f(x)| d \mu(t)+(S) \int_{[a, b]} f(x) d \mu(t),
$$

that is

$$
(S) \int_{[a, b]} f(t) d \mu(t)-(S) \int_{[a, b]} f(x) d \mu(t) \leq(S) \int_{[a, b]}|f(t)-f(x)| d \mu(t) .
$$

Similarly, we have

$$
f(x)=f(x)-f(t)+f(t) \leq|f(t)-f(x)|+f(t),
$$

then (by Theorem 2.3 (3) and Theorem 2.8)

$$
(S) \int_{[a, b]} f(x) d \mu(t) \leq(S) \int_{[a, b]}|f(t)-f(x)| d \mu(t)+(S) \int_{[a, b]} f(t) d \mu(t),
$$

that is

$$
(S) \int_{[a, b]} f(x) d \mu(t)-(S) \int_{[a, b]} f(t) d \mu(t) \leq(S) \int_{[a, b]}|f(t)-f(x)| d \mu(t) .
$$

By (8) and (9) we derive that

$$
\left|(S) \int_{[a, b]} f(t) d \mu(t)-(S) \int_{[a, b]} f(x) d \mu(t)\right| \leq(S) \int_{[a, b]}|f(t)-f(x)| d \mu(t) .
$$


Consequently it holds

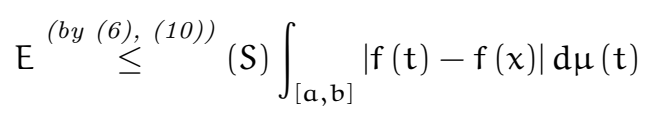

(and by $\left.|\mathrm{f}(\mathrm{t})-\mathrm{f}(\mathrm{x})| \leq\left\|\mathrm{f}^{\prime}\right\|_{\infty}|\mathrm{t}-\mathrm{x}|\right)$

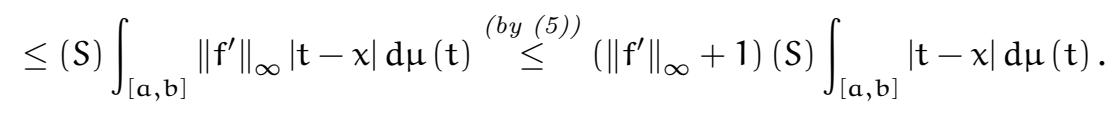

We have proved the following Ostrowski-like inequality

$$
\begin{gathered}
\left|\frac{1}{\mu([a, b])}(S) \int_{[a, b]} f(t) d \mu(t)-\frac{\mu([a, b] \wedge f(x))}{\mu([a, b])}\right| \leq \\
\frac{\left(\left\|f^{\prime}\right\|_{\infty}+1\right)}{\mu([a, b])}(S) \int_{[a, b]}|t-x| d \mu(t) .
\end{gathered}
$$

The last inequality can be better written as follows:

$$
\begin{gathered}
\left|\frac{1}{\mu([a, b])}(S) \int_{[a, b]} f(t) d \mu(t)-\left(1 \wedge \frac{f(x)}{\mu([a, b])}\right)\right| \leq \\
\frac{\left(\left\|f^{\prime}\right\|_{\infty}+1\right)}{\mu([a, b])}(S) \int_{[a, b]}|t-x| d \mu(t) .
\end{gathered}
$$

Notice here that $\left(1 \wedge \frac{\mathrm{f}(\mathrm{x})}{\mu([\mathrm{a}, \mathrm{b}])}\right) \leq 1$, and $\frac{1}{\mu([a, b])}(S) \int_{[a, b]} f(t) d \mu(t) \leq \frac{\mu([a, b])}{\mu([a, b])}=1$, where (S) $\int_{[a, b]} f(t) d \mu(t) \geq 0$.

I.e. If $\mathrm{f}:[\mathrm{a}, \mathrm{b}] \rightarrow \mathbb{R}_{+}$is a Lipschitz function of order $0<\alpha \leq 1$, i.e. $|\mathrm{f}(\mathrm{x})-\mathrm{f}(\mathrm{y})| \leq$ $\mathrm{K}|\mathrm{x}-\mathrm{y}|^{\alpha}, \forall x, y \in[\mathrm{a}, \mathrm{b}]$, where $\mathrm{K}>0$, denoted by $\mathrm{f} \in \operatorname{Lip}_{\alpha, \mathrm{K}}\left([\mathrm{a}, \mathrm{b}], \mathbb{R}_{+}\right)$, then we get similarly the following Ostrowski-like inequality:

$$
\begin{gathered}
\left|\frac{1}{\mu([a, b])}(S) \int_{[a, b]} f(t) d \mu(t)-\left(1 \wedge \frac{f(x)}{\mu([a, b])}\right)\right| \leq \\
\frac{(K+1)}{\mu([a, b])}(S) \int_{[a, b]}|t-x|^{\alpha} d \mu(t) .
\end{gathered}
$$

We have proved the following Ostrowski-Sugeno inequalities:

Theorem 3.2. Suppose that $\mu$ is a fuzzy subadditive measure with $\mu([a, b])>0, x \in[a, b]$.

1) Let $f \in C^{1}\left([a, b], \mathbb{R}_{+}\right)$, then

$$
\begin{gathered}
\left|\frac{1}{\mu([a, b])}(S) \int_{[a, b]} f(t) d \mu(t)-\left(1 \wedge \frac{f(x)}{\mu([a, b])}\right)\right| \leq \\
\frac{\left(\left\|f^{\prime}\right\|_{\infty}+1\right)}{\mu([a, b])}(S) \int_{[a, b]}|t-x| d \mu(t) .
\end{gathered}
$$


2) Let $f \in \operatorname{Lip}_{\alpha, \mathrm{K}}\left([\mathrm{a}, \mathrm{b}], \mathbb{R}_{+}\right), 0<\alpha \leq 1$, then

$$
\begin{gathered}
\left|\frac{1}{\mu([a, b])}(S) \int_{[a, b]} f(t) d \mu(t)-\left(1 \wedge \frac{f(x)}{\mu([a, b])}\right)\right| \leq \\
\frac{(K+1)}{\mu([a, b])}(S) \int_{[a, b]}|t-x|^{\alpha} d \mu(t) .
\end{gathered}
$$

We make the following remark

Remark 3.3. Let $\mathrm{f} \in \mathrm{C}^{1}\left([\mathrm{a}, \mathrm{b}], \mathbb{R}_{+}\right)$and $\mathrm{g} \in \mathrm{C}^{1}([\mathrm{a}, \mathrm{b}])$, by Cauchy's mean value theorem we get that

$$
(f(t)-f(x)) g^{\prime}(c)=(g(t)-g(x)) f^{\prime}(c),
$$

for some $\mathrm{c}$ between $\mathrm{t}$ and $\mathrm{x}$; for any $\mathrm{t}, \mathrm{x} \in[\mathrm{a}, \mathrm{b}]$.

If $\mathrm{g}^{\prime}(\mathrm{c}) \neq 0$, we have

$$
(f(t)-f(x))=\left(\frac{f^{\prime}(c)}{g^{\prime}(c)}\right)(g(t)-g(x)) .
$$

Here we assume that $\mathrm{g}^{\prime}(\mathrm{t}) \neq 0, \forall \mathrm{t} \in[\mathrm{a}, \mathrm{b}]$. Hence it holds

$$
|f(t)-f(x)| \leq\left\|\frac{f^{\prime}}{g^{\prime}}\right\|_{\infty}|g(t)-g(x)|,
$$

for all $t, x \in[a, b]$.

We have again as before (see (11))

$$
\begin{aligned}
& E \leq(S) \int_{[a, b]}|f(t)-f(x)| d \mu(t) \stackrel{(b y \leq ~[17))}{\leq} \\
& (S) \int_{[a, b]}\left\|\frac{f^{\prime}}{g^{\prime}}\right\|_{\infty}|g(t)-g(x)| d \mu(t) \stackrel{(b y) \text { (5)) }}{\leq} \\
& \left(\left\|\frac{f^{\prime}}{g^{\prime}}\right\|_{\infty}+1\right)(S) \int_{[a, b]}|g(t)-g(x)| d \mu(t) .
\end{aligned}
$$

We have established the following general Ostrowski-Sugeno inequality:

Theorem 3.4. Suppose that $\mu$ is a fuzzy subadditive measure with $\mu([a, b])>0, x \in[a, b]$. Let $\mathrm{f} \in \mathrm{C}^{1}\left([\mathrm{a}, \mathrm{b}], \mathbb{R}_{+}\right)$and $\mathrm{g} \in \mathrm{C}^{1}([\mathrm{a}, \mathrm{b}])$ with $\mathrm{g}^{\prime}(\mathrm{t}) \neq 0, \forall \mathrm{t} \in[\mathrm{a}, \mathrm{b}]$. Then

$$
\begin{gathered}
\left|\frac{1}{\mu([a, b])}(S) \int_{[a, b]} f(t) d \mu(t)-\left(1 \wedge \frac{f(x)}{\mu([a, b])}\right)\right| \leq \\
\frac{\left(\left\|\frac{f^{\prime}}{g^{\prime}}\right\|_{\infty}+1\right)}{\mu([a, b])}(S) \int_{[a, b]}|g(t)-g(x)| d \mu(t) .
\end{gathered}
$$


We give for $g(t)=e^{t}$ the next result

Corollary 3.5. Suppose that $\mu$ is a fuzzy subadditive measure with $\mu([a, b])>0, x \in[a, b]$. Let $f \in C^{1}\left([a, b], \mathbb{R}_{+}\right)$, then

$$
\begin{gathered}
\left|\frac{1}{\mu([a, b])}(S) \int_{[a, b]} f(t) d \mu(t)-\left(1 \wedge \frac{f(x)}{\mu([a, b])}\right)\right| \leq \\
\frac{\left(\left\|\frac{f^{\prime}}{e^{t}}\right\|_{\infty}+1\right)}{\mu([a, b])}(S) \int_{[a, b]}\left|e^{t}-e^{x}\right| d \mu(t) .
\end{gathered}
$$

When $g(t)=\ln t$ we get the following corollary.

Corollary 3.6. Suppose that $\mu$ is a fuzzy subadditive measure with $\mu([a, b])>0, x \in[a, b]$ and $\mathrm{a}>0$. Let $\mathrm{f} \in \mathrm{C}^{1}\left([\mathrm{a}, \mathrm{b}], \mathbb{R}_{+}\right)$. Then

$$
\begin{gathered}
\left|\frac{1}{\mu([a, b])}(S) \int_{[a, b]} f(t) d \mu(t)-\left(1 \wedge \frac{f(x)}{\mu([a, b])}\right)\right| \leq \\
\frac{\left(\left\|t f^{\prime}(t)\right\|_{\infty}+1\right)}{\mu([a, b])}(S) \int_{[a, b]}\left|\ln \frac{t}{x}\right| d \mu(t) .
\end{gathered}
$$

Many other applications of Theorem 3.4 could follow but we stop it here.

We make the following remark.

Remark 3.7. Let $\mathrm{f} \in\left[\mathrm{C}\left([\mathrm{a}, \mathrm{b}], \mathbb{R}_{+}\right) \cap \mathrm{C}^{\mathrm{n}+1}([\mathrm{a}, \mathrm{b}])\right], \mathrm{n} \in \mathbb{N}, x \in[\mathrm{a}, \mathrm{b}]$. Then by Taylor's theorem we get

$$
f(y)-f(x)=\sum_{k=1}^{n} \frac{f^{(k)}(x)}{k !}(y-x)^{k}+R_{n}(x, y)
$$

where the remainder

$$
R_{n}(x, y):=\int_{x}^{y}\left(f^{(n)}(t)-f^{(n)}(x)\right) \frac{(y-t)^{n-1}}{(n-1) !} d t ;
$$

here $\mathrm{y}$ can be $\geq \mathrm{x}$ or $\leq \mathrm{x}$.

By [1] we get that

$$
\left|R_{n}(x, y)\right| \leq \frac{\left\|f^{(n+1)}\right\|_{\infty}}{(n+1) !}|y-x|^{n+1}, \text { for all } x, y \in[a, b] .
$$

Here we assume $\mathrm{f}^{(\mathrm{k})}(\mathrm{x})=0$, for all $\mathrm{k}=1, \ldots, \mathrm{n}$.

Therefore it holds

$$
|f(t)-f(x)| \leq \frac{\left\|f^{(n+1)}\right\|_{\infty}}{(n+1) !}|t-x|^{n+1}, \text { for all } t, x \in[a, b] .
$$


Here we have again

$$
\begin{aligned}
E \leq(S) & \int_{[a, b]}|f(t)-f(x)| d \mu(t) \stackrel{\text { (by Theorem } \underset{2.3}{\leq} \text { (3) and (25)) }}{\leq} \\
& (S) \int_{[a, b]} \frac{\left\|f^{(n+1)}\right\|_{\infty}}{(n+1) !}|t-x|^{n+1} \mathrm{~d} \mu(t) \stackrel{(b y}{\leq} \text { (5)) } \\
& \left(\frac{\left\|f^{(n+1)}\right\|_{\infty}}{(n+1) !}+1\right)(S) \int_{[a, b]}|t-x|^{n+1} d \mu(t)
\end{aligned}
$$

We have derived the following high order Ostrowski-Sugeno inequality:

Theorem 3.8. Let $\mathrm{f} \in\left[\mathrm{C}\left([\mathrm{a}, \mathrm{b}], \mathbb{R}_{+}\right) \cap \mathrm{C}^{\mathrm{n}+1}([\mathrm{a}, \mathrm{b}])\right], \mathrm{n} \in \mathbb{N}, \mathrm{x} \in[\mathrm{a}, \mathrm{b}]$. We assume that $f^{(k)}(x)=0$, all $\mathrm{k}=1, \ldots, n$. Here $\mu$ is subadditive with $\mu([a, b])>0$. Then

$$
\begin{gathered}
\left|\frac{1}{\mu([a, b])}(S) \int_{[a, b]} f(t) d \mu(t)-\left(1 \wedge \frac{f(x)}{\mu([a, b])}\right)\right| \leq \\
\frac{\left(\frac{\left\|f^{(n+1)}\right\|_{\infty}}{(n+1) !}+1\right)}{\mu([a, b])}(S) \int_{[a, b]}|t-x|^{n+1} d \mu(t),
\end{gathered}
$$

which generalizes (15).

When $x=\frac{a+b}{2}$ we get the following corollary

Corollary 3.9. Let $\mathrm{f} \in\left[\mathrm{C}\left([\mathrm{a}, \mathrm{b}], \mathbb{R}_{+}\right) \cap \mathrm{C}^{\mathrm{n}+1}([\mathrm{a}, \mathrm{b}])\right], \mathrm{n} \in \mathbb{N}$. Assume that $\mathrm{f}^{(\mathrm{k})}\left(\frac{\mathrm{a}+\mathrm{b}}{2}\right)=0$, $\mathrm{k}=1, \ldots, \mathrm{n}$. Here $\mu$ is subadditive with $\mu([\mathrm{a}, \mathrm{b}])>0$. Then

$$
\begin{aligned}
& \left|\frac{1}{\mu([a, b])}(S) \int_{[a, b]} f(t) d \mu(t)-\left(1 \wedge \frac{f\left(\frac{a+b}{2}\right)}{\mu([a, b])}\right)\right| \leq \\
& \frac{\left(\frac{\left\|f^{(n+1)}\right\|_{\infty}}{(n+1) !}+1\right)}{\mu([a, b])}(S) \int_{[a, b]}\left|t-\frac{a+b}{2}\right|^{n+1} d \mu(t) .
\end{aligned}
$$




\section{References}

[1] G.A. Anastassiou, Ostrowski type inequalities, Proc. Amer. Math. Soc. 123(1995), 3775-3781.

[2] M. Boczek, M. Kaluszka, On the Minkowaki-Hölder type inequalities for generalized Sugeno integrals with an application, Kybernetica, 52(3) (2016), 329-347.

[3] A. Ostrowski, Über die Absolutabweichung einer differentiebaren Funktion von ihrem Integralmittelwert, Comment. Math. Helv., 10 (1938), 226-227.

[4] E. Pap, Null-Additive Set functions, Kluwer Academic, Dordrecht, 1995.

[5] D. Ralescu, G. Adams, The fuzzy integral, J. Math. Anal. Appl., 75 (1980), 562-570.

[6] M. Sugeno, Theory of fuzzy integrals and its applications, $\mathrm{PhD}$ thesis, Tokyo Institute of Technology (1974).

[7] Z. Wang, G.J. Klir, Fuzzy Measure Theory, Plenum, New York, 1992. 